\title{
Tratamento ao paciente queimado através do uso de enxerto de tilápia - uma revisão
}

\section{de literatura}

\author{
Treatment of the burned patient through the use of tilapia graft - a literature review \\ Tratamiento del paciente quemado mediante el uso de injerto de tilapia - revisión de la literatura
}

Recebido: 08/09/2021 | Revisado: 14/09/2021 | Aceito: 18/09/2021 | Publicado: 19/09/2021

\author{
Waldo Silva Mariz \\ ORCID: https://orcid.org/0000-0001-5995-8545 \\ Universidade Federal de Campina Grande, Brasil \\ E-mail:waldosilvamariz@gmail.com \\ Diego Costa Rodrigues \\ ORCID: https://orcid.org/0000-0002-9831-4192 \\ Universidade Federal de Campina Grande, Brasil \\ E-mail: diegocostar4@gmail.com \\ Jorge Pontual Waked \\ ORCID: https://orcid.org/0000-0002-2570-0228 \\ Universidade Federal de Campina Grande, Brasil \\ E-mail: jpwaked@gmail.com
}

\begin{abstract}
Resumo
Introdução: Pesquisas recentes apontam que a tilápia possui em sua pele uma microbiota não infecciosa que apresenta boa aderência ao leito das feridas, além de ser similar à estrutura morfológica presente na pele humana. Objetivo: Este estudo tem como objetivo demonstrar a eficácia da implantação da pele da tilápia como uma possível fonte de biomaterial para enxertia e curativo biológico para o tratamento de queimaduras. Métodos: O presente estudo é uma revisão de literatura exploratória. Para as buscas foram utilizados os bancos de dados: Biblioteca Virtual em Saúde, Scielo, Pubmed, LILACS e Medline. Foram utilizados como critérios de elegibilidade trabalhos publicados no período de 2004 a 2019 a partir das palavras-chave "tilápia" e "queimaduras". Foram excluídos os artigos que não se adequavam aos objetivos desta pesquisa. Resultados: As pesquisas mostraram que o perfil morfológico da pele da tilápia apresenta grande semelhança à da pele humana, com fibras colágenas dispostas na mesma consistência da derme profunda encontrada na pele humana, logo quando submetido ao tratamento aos pacientes queimados, a utilização da pele de tilápia mostrou uma recuperação quase $70 \%$ melhor como substituta do curativo convencional, além da diminuição do tempo de tratamento aos pacientes, com bons resultados. Conclusão: Com base na pesquisa pode-se concluir que a pele de tilápia traz uma grande promessa de um produto eficaz e inovador, de fácil aplicação e alta disponibilidade, havendo uma grande eficácia na cicatrização das queimaduras com diminuição de dores em pacientes.
\end{abstract}

Palavras-chave: Tilápia; Queimaduras; Colágeno.

\begin{abstract}
Introduction: Recent research indicates that tilapia has in its skin a non-infectious microbiota that presents good adherence to the wound bed, besides being similar to the morphological structure present in human skin. Objective: This study aims to demonstrate the efficacy of tilapia skin implantation as a possible source of biomaterial for grafting and biological dressing for the treatment of burns. Methods: The present study is a review of exploratory literature. For the searches, the following databases were used: Virtual Health Library, Scielo, Pubmed, LILACS and Medline. Papers published from 2004 to 2019 were used as eligibility criteria using the keywords "tilapia" and "burns". Articles that did not fit the objectives of this research were excluded. Results: Research has shown that the morphological profile of tilapia skin bears great resemblance to that of human skin, with collagen fibers arranged in the same consistency of the deep dermis found in human skin, soon when submitted to treatment to burned patients, the use of tilapia skin showed a recovery almost $70 \%$ better as a substitute for conventional dressing, in addition to reducing the treatment time to patients, with good results. Conclusion: Based on the research it can be concluded that tilapia skin brings a great promise of an effective and innovative product, easy to apply and high availability, with great efficacy in healing burns with decreased pain in patients.
\end{abstract}

Keywords: Tilapia; Burns; Collagen.

\section{Resumen}

Introducción: Investigaciones recientes indican que la tilapia tiene en su piel una microbiota no infecciosa que presenta buena adherencia al lecho de la herida, además de ser similar a la estructura morfológica presente en la piel humana. Objetivo: Este estudio tiene como objetivo demostrar la eficacia de la implantación de la piel de tilapia como 
una posible fuente de biomaterial para el injerto y el apósito biológico para el tratamiento de quemaduras. Métodos: El presente estudio es una revisión de la literatura exploratoria. Para las búsquedas se utilizaron las siguientes bases de datos: Biblioteca Virtual en Salud, Scielo, Pubmed, LILACS y Medline. Los trabajos publicados entre 2004 y 2019 se utilizaron como criterios de elegibilidad utilizando las palabras clave "tilapia" y "quemaduras". Se excluyeron los artículos que no se ajustaban a los objetivos de esta investigación. Resultados: La investigación ha demostrado que el perfil morfológico de la piel de la tilapia tiene un gran parecido con el de la piel humana, con fibras de colágeno dispuestas en la misma consistencia de la dermis profunda que se encuentra en la piel humana, pronto cuando se somete a tratamiento a pacientes quemados, el uso de piel de tilapia mostró una recuperación casi un $70 \%$ mejor como sustituto del apósito convencional, además de reducir el tiempo de tratamiento a los pacientes, con buenos resultados. Conclusión: Basado en la investigación se puede concluir que la piel de tilapia trae una gran promesa de un producto eficaz e innovador, fácil de aplicar y de alta disponibilidad, con gran eficacia en la curación de quemaduras con disminución del dolor en los pacientes.

Palabras clave: Tilapia; Quemaduras; Colágeno.

\section{Introdução}

Há de se notar que, no Brasil, aproximadamente dois milhões de pessoas ao ano apresentam queimadura, a qual ocorre devido a um trauma de origem térmica e comumente se apresenta desde bolhas menores até situações mais complexas variando em extensão e profundidade, visto que depende da resposta que o corpo desencadeia frente ao estímulo (2004; Júnior, Moscozo, Filho, De Menezes et al., 2007). O tratamento destes pacientes que se encontram com queimadura na rede pública brasileira é feito principalmente através do tratamento local por meio da pomada sulfadiazina de prata, porém não é suficiente para solucionar em sua totalidade as diversas lesões provenientes da queimadura. É válido ressaltar que o tratamento tem sido enfrentado como um desafio aos profissionais de todo o mundo, seja pela exigência de um tratamento intensivo em certos casos, ou pela complexidade das lesões e a necessidade de um tratamento multidisciplinar (Júnior, 2017; Júnior, Moscozo, Filho, De Menezes et al., 2007).

Em alguns países da América do Sul, nos Estados Unidos e até na Europa o aloenxerto realizado a partir da pele humana é eficaz para o tratamento de queimaduras. Entretanto, a partir de dados fornecidos pelo Ministério da Saúde, é possível ressaltar que o Brasil tem 13 bancos de pele, porém somente quatro funcionam e o desempenho destes se encontra longe de suprir a carência (Júnior, 2019; Lima Júnior, Barreto, 2009). O aloenxerto enfrenta dificuldades de se concretizar no Brasil, principalmente pela pouca quantidade de pele humana disponível em hospitais públicos e pelo alto custo da produção (Júnior, 2019). Diante dessa situação, outros materiais biológicos têm surgido como uma alternativa promissora para esse tratamento, visto que a maioria das vítimas são diagnosticadas com queimaduras de segundo grau (Batista, Cordovil, 2012; Brasil. Ministério Da Saúde. Secretaria De Atenção À Saúde. Departamento De Atenção Especializada; De Miranda, Brandt, 2019).

Diante disso, o xenoenxerto é uma importante alternativa a esse tratamento. Assim, um grupo de pesquisadores desenvolveu um projeto de pesquisa sobre o uso da pele do peixe tilápia para o tratamento de queimaduras, funcionando como um curativo biológico oclusivo (Júnior, 2019).

A tilápia é o peixe mais cultivado do Brasil e um dos mais cultivados do mundo, apresenta uma microbiota não infecciosa, é abundante em nossa fauna e a indústria da pesca pouco aproveita a pele deste animal. A estrutura morfológica desta é similar à da pele humana e, de acordo com estudos histológicos, possui consideráveis quantidades de colágeno tipo 1, chegando ser até maiores que as encontradas em pele humana. Com base nisso, o Brasil já possui registro de pele animal na Agência Nacional de Vigilância Sanitária (ANVISA) e esta é encontrada disponível no Sistema Único de Saúde (SUS) para ser utilizada em pacientes queimados (Alves, Júnior; Piccolo, De Miranda et al., 2018; Alves, Verde, Ferreira Júnior, Silva et al., 2015; Junior, Bandeira, Miranda, Ferreira et al., 2016; Júnior, 2017; Oliveira, 2016).

Vale ressaltar também que os resultados experimentais da pele da tilápia para o tratamento de queimaduras em ratos foram excelentes e estudos histológicos constataram que a pele da tilápia apresentou boa aderência à ferida e uma consequente 
melhora na cicatrização, sem alterações consideráveis na estrutura da derme (Júnior, 2017; Lima-Junior, Picollo; Miranda, Ribeiro et al., 2017). Este estudo tem como objetivo demonstrar a eficácia da implantação da pele da tilápia como uma possível fonte de biomaterial para enxertia e curativo biológico no tratamento de queimaduras.

\section{Metodologia}

Segundo Pereira et al. (2018), as revisões bibliográficas devem especificar a origem dos dados abordados, o intervalo pesquisado e os métodos utilizados. Dessa forma, o presente estudo é uma revisão de literatura exploratória que aborda um retrato sobre a eficácia da pele de tilápia ao leito da ferida e sua cicatrização, através de buscas de suas variáveis, para se detectar sua frequência de utilização e eficácia. Para as buscas foram utilizados os bancos de dados: BVS Brasil (Biblioteca Virtual em Saúde), Scielo (Scientific Eletronic Library Online), Pubmed (National Center for Biotechnology Information), LILACS (Literatura Latino-americana e do Caribe em Ciências da Saúde) e Medline. Foram utilizados como critérios de elegibilidade trabalhos publicados no período de 2004 a 2019 a partir das palavras-chave "tilápia" e "queimaduras". Os artigos selecionados foram lidos na íntegra. Foram excluídos os artigos que não se adequavam aos objetivos desta pesquisa. Ao final, foram obtidos 25 artigos para serem utilizados na elaboração do presente estudo.

\section{Resultados e Discussão}

\section{Banco de pele animal do Brasil}

Há de se notar que a cultivação e o incentivo da utilização da pele da tilápia está diretamente relacionada ao Banco de Pele Animal Aquático, dessa forma, para a efetuação do Banco de Pele Animal é necessário que várias etapas sejam cumpridas. Os processos consistem em estudar o cativeiro, montar o laboratório de cicatrização, realizar estudos pré-clínicos e fazer os registros de patentes, reconhecer a área física para montagem do Banco de Pele Animal, visitar bancos de pele humana, exercer a presença de consultoria técnica, estabelecer o processo de esterilização e cumprir o registro e a padronização de todos os processos de produção da pele de tilápia (Júnior, 2019).

Durante o processo de distribuição da pele da tilápia para estudos, são realizadas diversas pesquisas nas mais diversas áreas como: ginecologia, ortopedia, endoscopia, estomaterapia, cirurgia vascular, odontologia e veterinária. Desta forma, é possível encontrar casos clínicos e situações em que a utilização desta pele pode ocorrer nas lesões por queimaduras, nas reconstruções de vagina, nas úlceras varicosas, nas feridas por pressão, nas feridas em cães, além de estudos experimentais em animais. Devido a essas práticas realizarem atividades com um ou mais tipos de tecidos de origem humana com finalidade terapêutica, para validar e garantir a qualidade dos tecidos fornecidos é necessário seguir as diretrizes mediadoras exigidas pela Agência Nacional de Vigilância Sanitária (ANVISA) (Ministério da Saúde (BR). Agência Nacional de Vigilância Sanitária (ANVISA). Resolução da diretoria colegiada - RDC n 55, de 11 de dezembro de 2015. Dispõe sobre as Boas Práticas em Tecidos humanos para uso terapêutico. Diário Oficial da União, Brasília (DF). 2015; Ministério da Saúde (BR). Agência Nacional de Vigilância Sanitária (ANVISA). Relatório De Avaliação Dos Dados De Produção Dos Bancos De Tecidos - Ano 2020, Brasil. Brasília (Df): Anvisa, 2020; Júnior, 2019).

O Banco de Pele Animal Aquático já processou milhares de peles de tilápia do Nilo (Oreochromis niloticus). Logo, se apresenta como uma alternativa eficaz, protegendo o leito da ferida contra perdas de líquido e de calor, infecções bacterianas, reduzindo a dor e estimulando a cicatrização, além de ser importante para diminuir a necessidade de pele humana no país para o tratamento de queimados, algo que vem sendo comprovado com resultados positivos e que evidenciam a importância e a segurança desse tecido (Júnior, 2019). 


\section{Estudo laboratorial e de toxicidade}

Segundo pesquisas (Alves, Verde, Ferreira Júnior, Silva et al., 2015; Júnior, 2017; Oliveira, 2016), a reepitelização da queimadura do paciente se dá geralmente em 16 dias e não ocorrem efeitos colaterais, evidenciando que a pele da tilápia não possui antigenicidade e toxicidade. Outrossim, é capaz de manter a umidade, reduzir o trabalho da equipe e dos custos e realizar uma boa aderência ao leito da ferida, promovendo uma reepitelização e facilitando a formação do tecido de granulação. Dessa forma, a utilização da pele da tilápia contribui para escapar dos riscos de se infectar externamente pela entrada de microrganismos, além de diminuir a perda de líquidos e não ser necessária sua extração (Alves, Verde, Ferreira Júnior, Silva et al., 2015; Chiu, Burd, 2005; De Miranda, 2018; Júnior, 2017).

As peles da Tilápia-do-Nilo passam por processos criteriosos nos quais estas são descontaminadas (clorexidina a $2 \%$ e glicerol em altas concentrações) e esterilizadas (irradiação com gama cobalto 60) com o intuito de ser segura a sua utilização. Esses cuidados são necessários para a realização do controle de micro-organismos, pois eles podem atrasar a cicatrização. Na etapa de laboratório de cicatrização, os tecidos disponibilizados para serem utilizados são estocados na geladeira, com temperaturas que variam entre 2 e $4{ }^{\circ} \mathrm{C}$ e possuem validade de até dois anos. De acordo com os resultados obtidos pode-se afirmar que a pele da Tilápia-do-Nilo é eficaz como curativo biológico oclusivo no manejo/tratamento de queimaduras (De Miranda, Brandt, 2019; Júnior, 2019; Pereima, Rodrigues, Lopes, 2013).

\section{Estudo histológico}

De acordo com a literatura (Barone, Mastroianni, Farkash, Mallard et al., 2015), alguns estudos demonstraram por análises histológicas que a epiderme da pele de tilápia é revestida por um epitélio pavimentoso estratificado. As células mais profundas são basais e apresentam forma colunar, já as células mais superficiais apresentam formas espinhosas, poligonais com núcleos ovais (Barone, Mastroianni, Farkash, Mallard et al., 2015). Outrossim, em relação à constituição do tecido em aspectos mais profundos, a derme mais superficial é quase toda composta por tecido conjuntivo frouxo envolto por vasos sanguíneos de diferentes calibres, com fibras paralelas de tecido colágeno e menos espessa, além de melanóforos subepiteliais. Já na derme mais profunda, encontram-se grossas fibras colágenas bem mais organizadas, compactas, de disposição horizontal, perpendiculares à superfície da pele e possuindo algumas semelhanças à pele de outros animais, inclusive humanos (Alves, Verde, Ferreira Júnior, Silva Et Al., 2015; Barone, Mastroianni, Farkash, Mallard et al., 2015).

Uma pesquisa brasileira (Franco, Franco, Gasparino, Dorado et al., 2013), cujo intuito era caracterizar a pele de tilápia a partir de suas características histomorfológicas e de tipificação, demonstrou que a similaridade da composição da pele de tilápia comparada à da pele humana, principalmente nos tipos de colágenos, apresentou quase $98 \%$ de efetividade e compatibilidade. Dessa forma, a utilização da pele da tilápia em humanos é viável como curativo biológico de qualidade para o tratamento de queimaduras em humanos (Alves, Verde, Ferreira Júnior, Silva Et Al., 2015; Franco, Franco, Gasparino, Dorado Et Al., 2013). Além da eficácia na cicatrização, também foi constatado ausência de germes gram (+), gram (-) e fungos a na pele de tilápia, e nenhuma alteração histológica na estrutura da derme e seus elementos. Dessa forma, os riscos de infecções por agentes bacterianos ao contato do tecido humano tornam-se muito baixos, mostrando grande efetividade da tilápia como agente curativo e cicatrizante (Junior, Bandeira, Miranda, Ferreira et al., 2016).

\section{Os requisitos e benefícios quanto à utilização da pele da tilápia}

Para o tratamento da ferida por queimadura, cuidados locais e sistêmicos devem ser tomados, variando de acordo com profundidade, localização corporal e extensão da lesão (Junior, Bandeira, Miranda, Ferreira Et Al., 2016; Oliveira, 2016). A pele queimada é susceptível à contaminação, devendo o curativo biológico apresentar propriedades que evitem proliferação microbiana de acordo com todos os manuseios de biossegurança (Alves, Verde, Ferreira Júnior, Silva Et Al., 2015). Para o seu 
uso como curativo oclusivo, faz-se necessário um rigoroso protocolo de desinfecção e esterilização que não altere suas propriedades microscópicas e tensiométricas (Junior, Bandeira, Miranda, Ferreira et al., 2016). Pesquisa recente indica que o uso de esterilização química e radio esterilização em concomitância é efetivo para o preparo da pele de Tilápia do Nilo (Alves, Verde, Ferreira Júnior, Silva et al., 2015). Nos centros privados de tratamento de queimados do Hospital José Frota em Fortaleza já são utilizadas peles da tilápia como curativos biossintéticos e peles artificiais, que são de elevado custo. Por outro lado, no setor público da Universidade Federal do Ceará, os centros realizam desbridamento sequencial, uso de antimicrobiano tópico e preparo do leito para utilizar a pele da tilápia como enxerto com menores custos (Alves, Júnior, Piccolo, De Miranda et al., 2018; Junior, Bandeira, Miranda, Ferreira et al., 2016).

O curativo utilizando a pele de tilápia resulta em proteção do ferimento e diminui a necessidade de inúmeras trocas de curativos devido ao fato de a sua remoção não ser necessária, permanecendo na ferida até a sua completa cicatrização e proliferação celular (Ministério da Saúde (BR). Agência Nacional de Vigilância Sanitária (ANVISA). Resolução da diretoria colegiada - $\mathrm{RDC} \mathrm{n}^{\circ}$ 55, de 11 de dezembro de 2015). Dispõe sobre as Boas Práticas em Tecidos humanos para uso terapêutico. (Diário Oficial Da União, Brasília (Df). 2015; Lima-Junior, Picollo, Miranda, Ribeiro et al., 2017). A análise do número de analgesias comparativas em terapia convencional com sulfadiazina de prata dos hospitais tradicionais demonstrou que a utilização de pele de tilápia diminuiu o uso de analgésicos para redução da dor em 20 vezes nos pacientes dos centros de queimados e reabilitação (Lima-Junior, Picollo, Miranda, Ribeiro et al., 2017). A redução da dor no manejo dos pacientes queimados é um resultado desejado por todos os profissionais envolvidos (Ministério da Saúde (BR). Agência Nacional de Vigilância Sanitária (ANVISA). Relatório de Avaliação dos Dados de Produção dos Bancos de Tecidos - Ano 2020, Brasil. Brasília (DF): ANVISA, 2020). Cada peixe fornece duas peles de 15 centímetros $(\mathrm{cm})$ por $05 \mathrm{~cm}$, e a quantidade de pele necessária para o curativo é dependente da extensão da lesão (Ministério da Saúde (BR). Agência Nacional de Vigilância Sanitária (ANVISA). Relatório de Avaliação dos Dados de Produção dos Bancos de Tecidos - Ano 2020, Brasil. Brasília (DF): ANVISA; 2020). A produção de tilápia no Brasil é viável para uso medicinal, desde que o processo de preparo seja produzido em escala industrial para o benefício social (Lima-Junior, Picollo, Miranda, Ribeiro et al., 2017). Várias especialidades em saúde já iniciaram pesquisas para a utilização da pele de tilápia como opção de tratamento de enfermidades além das queimaduras, como urologia, odontologia e ginecologia, sendo cada vez mais promissor ao futuro da medicina (De Miranda, Brandt, 2019; Junior, Bandeira, Miranda, Ferreira et al., 2016; Oliveira, 2016).

\section{Conclusão}

De acordo com a análise dos trabalhos, pode-se concluir que a pele de tilápia traz uma grande promessa de um produto eficaz e inovador, de fácil aplicação, com alta disponibilidade e não possui antigenicidade e toxicidade. Se caracteriza como um importante curativo flexível e aderente, que evita crescimentos microbianos, promove a reepitelização e facilita a formação do tecido de granulação. A maior parte da composição do colágeno da pele de tilápia é de colágeno tipo I, mostrando grande semelhança com a de humano e possuindo o poder de melhores recuperação e cicatrização nas queimaduras em humanos. Demonstra ausência de germes gram (+), gram (-), fungos, e alterações histológicas na estrutura da derme e seus elementos. Além disso, há grande eficácia para o tratamento de queimaduras, promovendo completa cicatrização e diminuição de dores.

\section{Referências}

Alves, A. P. N. N., Júnior, E. M. L., Piccolo, N. S., de Miranda, M. J. B., Verde, M. E. Q. L., Júnior, A. E. C. F., \& de Moraes, M. O. (2018). Study of tensiometric properties, microbiological and collagen content in nile tilapia skin submitted to different sterilization methods. Cell and tissue banking, 19(3), 373-382. 
Alves, A. P. N. N., Verde, M. E. Q. L., Ferreira Júnior, A. E. C., Silva, P. G. D. B., Feitosa, V. P., Lima Júnior, E. M., \& Moraes Filho, M. O. D. (2015). Avaliação microscópica, estudo histoquímico e análise de propriedades tensiométricas da pele de tilápia do Nilo. Revista Brasileira de Queimaduras, 14(3), 203-210.

Barone, A. A. L., Mastroianni, M., Farkash, E. A., Mallard, C., Albritton, A., Torabi, R., \& Cetrulo Jr, C. L. (2015). Genetically modified porcine splitthickness skin grafts as an alternative to allograft for provision of temporary wound coverage: preliminary characterization. Burns, $41(3)$, $565-574$.

Batista, B. D. F. C., \& Cordovil, P. B. (2012). Perfil epidemiológico de pacientes que sofreram queimaduras no Brasil: revisão de literatura. Revista Brasileira de Queimaduras, 11(4), 246-250.

Básica, A. (2013). Ministério Da Saúde Secretaria De Atenção À Saúde Departamento De Atenção Básica. Saúde Mais Perto De Você-Acesso E Qualidade; Programa Nacional De Melhoria Do Acesso E Da Qualidade Da Atenção Básica (Pmaq-Ab); Certificação Das Equipes De Atenção Básica; Participantes Do PMAQ, http://189.28.128.100/dab/docs/portaldab/documentos/certificacao_equipes_lista6.pdf.

Chiu, T., \& Burd, A. (2005). "Xenograft” dressing in the treatment of burns. Clinics in dermatology, 23(4), 419-423.

De Miranda, M. J. B. (2018). Viabilidade da pele de Tilápia do Nilo (Oreochromis niloticus) como curativo biológico no tratamento de queimaduras. Anais da faculdade de medicina de olinda, 1(1), 49-52.

De Miranda, M. J. B., \& Brandt, C. T. (2019). Xenoenxerto (pele da Tilápia-do-Nilo) e hidrofibra com prata no tratamento das queimaduras de II grau em adultos. Rev. bras. cir. plást, 79-85.

Guirro, E. C. D. O. F., \& Guirro, R. R. D. J. F. (2004). Dermato-funcional: fundamentos, recursos, patologias. Queimaduras, $491-497$.

Franco, M. L. R. S., Franco, N. P., Gasparino, E., Dorado, D. M., Prado, M., \& Vesco, A. P. D. (2013). Comparação das peles de tilápia do Nilo, pacu e tambaqui: Histologia, composição e resistência. Archivos de zootecnia, 62(237), 21-32.

Junior, E. M. L., Bandeira, T. D. J. P. G., de Miranda, M. J. B., Ferreira, G. E., Parente, E. A., Piccolo, N. S., \& de Moraes Flho, M. O. (2016). Characterization of the microbiota of the skin and oral cavity of Oreochromis niloticus. Journal of Health \& Biological Sciences, 4(3), $193-197$.

Júnior, E. M. L. (2017). Tecnologias inovadoras: uso da pele da tilápia do Nilo no tratamento de queimaduras e feridas. Rev Bras Queimaduras, 16(1), 1-2.

Júnior, E. M. L., Moraes-Filho, M. O., Rocha, M. B. S., Silva-Júnior, F. R., Philopimin, C. M., \& DO, M. F. A. (2019). Elaboração, desenvolvimento e instalação do primeiro banco de pele animal no Brasil para o tratamento de queimaduras e feridas. Rev. Bras. Cir. Plást., 34, 349-354.

Júnior, J. B. G., Moscozo, M. V. A., Filho, A. L. L., \& Gomes, C. M. G. (2007). Tratamento de pacientes queimados internados em hospital geral. Rev. Soc. Bras. Cir. Plást, 22(4), 228-32.

Lima Júnior, E. M., \& Barreto, M. G. P. (2009). Rotina de atendimento ao queimado. Gráfica LCR, 62(2), 312-6.

Lima-Junior, E. M., Picollo, N. S., Miranda, M. J. B. D., Ribeiro, W. L. C., Alves, A. P. N. N., Ferreira, G. E., \& Moraes Filho, M. O. D. (2017). Uso da pele de tilápia (Oreochromis niloticus), como curativo biológico oclusivo, no tratamento de queimaduras. Revista brasileira de queimaduras, $16(1), 10-7$.

Ministério da Saúde (BR), (2020). Agência Nacional de Vigilância Sanitária (ANVISA). Relatório de Avaliação dos Dados de Produção dos Bancos de Tecidos - Brasil. Brasília (DF): ANVISA, 1-8.

Ministério da Saúde (BR), (2015). Agência Nacional de Vigilância Sanitária (ANVISA). Resolução da diretoria colegiada - RDC n 55, de 11 de dezembro de 2015. Dispõe sobre as Boas Práticas em Tecidos humanos para uso terapêutico. Diário Oficial da União, Brasília (DF).

Oliveira, M. (2016). Tilapia’s turn. Pesquisa FAPESP, 249, 66-71.

Pereira, A. S., Shitsuka, D. M., Parreira, F. J., \& Shitsuka, R. (2018). Metodologia da pesquisa científica. UFSM, 1, 1-119.

Pereira, H. M., Rodrigues, R., \& Lopes, M. J. (2013). Evolução dos curativos de prata no tratamento de queimaduras de espessura parcial. Revista Brasileira de queimaduras, 12(2), 60-67. 